\title{
Modified Transition State Theory and Negative Apparent Activation Energies of Simple Metathesis Reactions: Application to the Reaction $\mathrm{CH}_{3}+\mathrm{HBr} \rightarrow \mathrm{CH}_{4}+\mathrm{Br}^{\dagger}$
}

\author{
Lev N. Krasnoperov, ${ }^{*, \sharp}$ Jingping Peng, ${ }^{\S}$ and Paul Marshall ${ }^{\S}$ \\ Department of Chemistry and Environmental Science, New Jersey Institute of Technology, \\ Newark, New Jersey 07102, and Department of Chemistry, University of North Texas, \\ P.O. Box 305070, Denton, Texas 76203-5070
}

Received: August 8, 2005; In Final Form: October 5, 2005

\begin{abstract}
A modified transition state theory (MTST) has been developed for gas-phase reactions with "negative barriers". The theory was applied to the reactions $\mathrm{CH}_{3}+\mathrm{HBr}(\mathrm{DBr}) \rightarrow \mathrm{CH}_{4}\left(\mathrm{CH}_{3} \mathrm{D}\right)+\mathrm{Br}(1 \mathrm{a}, 1 \mathrm{~b})$, which exhibit negative temperature dependences. Accurate ab initio calculations performed with coupled cluster theory extrapolated to the complete basis set limit revealed a transition state located at $-2.3 \mathrm{~kJ} \mathrm{~mol}^{-1}$ relative to the ground state of the reactants (in reaction 1a), as well as a shallow bound complex. The negative temperature dependence, the absolute values of the rate constant, and the isotope substitution effect are reproduced with good accuracy $(10 \%)$, without any adjustment or fitting parameters. Analytical expressions are presented for MTST including angular momentum conservation, centrifugal barriers and tunneling. This analysis uses information about the possibly loose entrance barrier and the transition state but does not invoke a statistical intermediate complex.
\end{abstract}

\section{Introduction}

Negative temperature dependence (negative apparent activation energies) in bimolecular reactions is a well-established phenomenon. ${ }^{1-7}$ Typically, negative temperature dependence is exhibited by reactions that proceed via formation of a bound long-lived statistical complex, separated from the reactants and the products by transition states. An explanation of the phenomenon was given on the basis of RRKM theory applied to the unimolecular dissociation of the statistical complex. Extended discussion as well as a theory of such reactions was provided by Mozurkewich and Benson. ${ }^{8}$ Negative apparent activation energies are normally expected when the transition state that leads to the products of the reaction has an energy below that of the reactants. Benson and Dobis ${ }^{9}$ reviewed the mechanism of reactions proceeding via an intermediate complex and discussed the major features such as possible pressure dependence, the inverse isotope substitution effect, and possible inversion of the negative temperature dependence to a positive one at elevated temperatures. Essentially, the major features of these reactions are well understood in terms of the multistep mechanism. ${ }^{10}$

During the last two decades, negative apparent activation energies were observed in bimolecular reactions that had been assumed to be "simple methathesis reactions". These are reactions of $\mathrm{C}$ - and $\mathrm{Si}$-centered small free radicals with hydrogen halides (HX, X $=\mathrm{I}, \mathrm{Br})$ or halogens $\left(\mathrm{X}_{2}\right) .{ }^{1,2,11-14}$ These studies had a significant impact on the derived thermochemistry of small hydrocarbon free radicals and the strength of the $\mathrm{C}-\mathrm{H}$ bonds in hydrocarbons. ${ }^{1,2,11}$ These new findings were met with scepticism due to the long-time belief in the nature of these

\footnotetext{
† Part of the special issue "Jürgen Troe Festschrift".

* To whom correspondence should be addressed. Fax: (973)-596-3586. E-mail: krasnoperov@adm.njit.edu.

$\doteqdot$ New Jersey Institute of Technology.

$\S$ University of North Texas.
}

reactions as "simple metathesis" (ref 15 and references therein). Tschuikow-Roux and co-workers ${ }^{16,17}$ attempted to explain the negative apparent activation energy for the reaction of $\mathrm{CH}_{3}$ with $\mathrm{HBr}$ based on the theory developed by Mozurkewich and Benson ${ }^{10}$ and quantum chemical calculations of the potential energy surface for the reaction. The theoretical calculations identified a weakly bound van der Waals $\mathrm{H}_{3} \mathrm{C}-\mathrm{H}-\mathrm{Br}$ complex at the entrance of the reactant valley. However, the potential well obtained in the calculations was very shallow (ca. $1 \mathrm{~kJ}$ $\mathrm{mol}^{-1}$ ), which makes the notion of a "statistical complex" somewhat doubtful. Moreover, the energy barrier as well as the predicted apparent activation energy were still positive, and to fit the experimental data the barrier in $\mathrm{CH}_{3}+\mathrm{HBr}$ was forced down by $3.3 \mathrm{~kJ} \mathrm{~mol}^{-116}$ whereas almost no adjustment was required for $\mathrm{CH}_{3}+\mathrm{HCl}$, which was a subject of further criticism. ${ }^{15}$

In this work, two major advances toward the understanding of the negative temperature dependence of simple metathesis reactions are made. First, the discussion is based on (modified) transition state theory (TST) rather than the RRKM theory, so that the requirement of a statistical complex is not needed anymore. However, although the same basic assumptions of TST are used, a modification, similar to that made in the Improved Canonical Variational Theory (ICVT) of Garrett and Truhlar, ${ }^{18-20}$ is required to describe systems properly where the ground state of the TS lies below the reactants level. A theory that takes into account a "negative barrier", conservation of angular momentum, the centrifugal barrier at the entrance of the reaction valley, and the quantum effects of tunneling and above-thebarrier reflection was developed and formulated in terms of analytical expressions. Second, investigation of the potential energy surface (PES) computed at a high level resulted in a negative energy of the TS. Calculations performed using the modified TST with the PES obtained in the new theoretical calculations predict the temperature dependence, the absolute value of the rate constant, as well as the isotope substitution 
effect in reaction 1 without any adjustments or fitting parameters.

$$
\begin{gathered}
\mathrm{CH}_{3}+\mathrm{HBr} \rightarrow \mathrm{CH}_{4}+\mathrm{Br} \\
\mathrm{CH}_{3}+\mathrm{DBr} \rightarrow \mathrm{CH}_{3} \mathrm{D}+\mathrm{Br}
\end{gathered}
$$

There have been several prior computational investigations of the PES for reaction 1. Issues arising from these studies include the possible existence of a bound intermediate, whether the energy of the TS is above or below that of the reactants, the correct analysis to derive rate constants, and the role of quantum mechanical tunneling.

The pioneering studies by Chen et al. were based on Gaussian-1 theory. ${ }^{21}$ As noted above, they computed a positive barrier to reaction which had to be adjusted to a negative value to match experiment, and they included a simple tunneling correction. Both corrections were criticized by Benson and Dobis, ${ }^{22}$ who argued in favor of smaller $\mathrm{CH}_{3}+\mathrm{HBr}$ rate constants with a positive activation energy. Yu and Nyman ${ }^{23}$ conducted quantum scattering calculations, which inherently treat tunneling correctly, based on a PES derived with MP2 second-order perturbation theory that yields a positive barrier. Guha and Francisco applied quadratically converged configuration interaction. ${ }^{24}$ Their highest level QCISD(T)/6-311++G(3df,3pd) results yielded $\Delta_{\mathrm{r}} H_{0}$ of $-55.6 \mathrm{~kJ} \mathrm{~mol}^{-1}$, in moderate accord with the experimental value of $-69.4 \mathrm{~kJ} \mathrm{~mol}^{-1}{ }^{25}$ Because of uncertainties in their energetics, they regarded the slightly negative reaction barrier they obtained with caution, and concluded that there is "essentially no barrier". EspinosaGarcia applied coupled cluster $\operatorname{CCSD}(\mathrm{T})$ theory with a triple- $\zeta$ basis set. ${ }^{26} \mathrm{He}$ argued that the reaction complex was an artifact of basis set superposition error, and that the reaction barrier is positive. The most recent study is that by Sheng et al., ${ }^{27}$ who applied spin-projected MP4 theory at QCISD/6-31+G(d) geometries to obtain $\Delta_{\mathrm{r}} H_{0}$ of $-59.8 \mathrm{~kJ} \mathrm{~mol}^{-1}$ and energies below reactants for the complex and TS. The latter two PESs were analyzed with improved canonical variational theory. ${ }^{18-20}$ Good agreement (within 20\%) with the experimental data was achieved. The new PES calculations here use $\operatorname{CCSD}(\mathrm{T})$ theory for high accuracy, with basis sets extrapolated to the infinite basis limit. At this limit any BSSE is eliminated. The PES should be more accurate than previous ones, and in particular enables definitive identification of a bound complex and a negative TS energy.

\section{Modified Transition State Theory for Reactions with "Negative Barriers"}

The transition state theory ${ }^{28,29}$ and its further developments are discussed in detail in numerous original publications and textbooks. ${ }^{28-46}$ For reactions with "negative barriers", i.e., when the ground state in the bottleneck position lies below the ground state of the reactants, a modification that takes into account nonavailability of the negative total energies should be introduced, such as was done by Garrett and Truhlar in their Improved Canonical Variational Theory. ${ }^{18-20}$ Calculations using variational transition state theories in general are more elaborate and require much more information about the potential energy surface than the original transition state theory where the critical surface is prescribed and only the properties of the "transition state" are required. Therefore, the traditional TST approach was extended to reactions with "negative barriers". The main expressions for the modified TST are derived below.

Notations. The discussion is focused on a bimolecular reaction

$$
\mathrm{A}+\mathrm{B} \rightarrow(\mathrm{AB})^{\ddagger} \rightarrow \text { products }
$$

In the discussion below, we will deal with $J, K$ specific rate constants. To avoid some difficulties associated with the separation of the partition function for "external rotation" of the pair of reactant molecules and with the treatment of the K-rotor as a degree of freedom with conserved energy, an artificial unbound "complex" between the reactants and the transition state is introduced. This reduces the problem to unimolecular reaction from the complex to the products. The complex could be a nonbonded artificial construct of the reactant molecules separated by a distance $r$ within a layer $\delta$, so that the rotational constants of the "complex" are well defined. The complex is assumed to be in microcanonical equilibrium (not the steady state) with the reactants. It is easy to show that the properties (the partition function) of this complex will cancel out in any derivation based on an equilibrium theory; therefore the precise specification of the parameters of such complex are not required (Appendix 1). Though it is not critical, for simplicity it is assumed that the energy of the ground state of the "complex" is the same as the ground state energy of the reactants. In the derivation below, the results will be obtained under the assumption of vanishing rotational constant $B$ of the complex, $B \rightarrow 0$, and the rotational constant $A$ equal to that of the transition state $\left(A=A^{\ddagger}\right)$.

The translational motion of the center-of-mass is factored out in all expressions and derivations below unless stated otherwise. The notations used for the partition functions, the densities and the number of states are listed below. All partition functions that include translational motion are per unit volume, all partition functions that do not include translational motion are dimensionless, and the units of the partition functions (based on $\mathrm{cm}^{3}$ ) are shown.

- $\mathrm{Q}_{\mathrm{A}}\left(\mathrm{cm}^{-3}\right) \mathrm{Q}_{\mathrm{B}}\left(\mathrm{cm}^{-3}\right), \mathrm{Q}_{\mathrm{C}}\left(\mathrm{cm}^{-3}\right), \mathrm{Q}_{\text {total }}^{\ddagger}\left(\mathrm{cm}^{-3}\right)$ are the total (including translational motion of the center of mass) partition functions of reactants A, B, the complex, and the total partition function of the transition state.

- $\mathrm{Q}_{\mathrm{R}}\left(\mathrm{cm}^{-3}\right)$ is the partition function of the reactants with the center-of-mass motion factored out, so that $\mathrm{Q}_{\mathrm{R}}=\mathrm{Q}_{\mathrm{A}} \mathrm{Q}_{\mathrm{B}} / \mathrm{Q}^{(3)}{ }_{t r, \mathrm{M}}$,

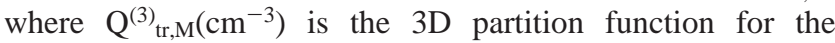
translational motion of the center of mass and $M=M_{A}+M_{B}$ is the total mass of $\mathrm{A}+\mathrm{B}$.

$\bullet \mathrm{Q}, \mathrm{Q}^{\ddagger}$ (dimensionless) are the partition functions (including external rotations) of the "complex" and the transition state with the translational motion of the center of mass factored out, so

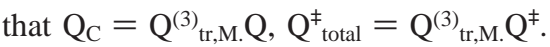

$-\mathrm{Q}_{\text {rot }}, \mathrm{Q}_{\text {rot }}^{\ddagger}$ (dimensionless) are the partition functions for external rotations of the "complex" and the transition state. Throughout the derivations the notations for the symmetric top are used, where all external rotations are characterized by three quantum numbers, $\mathrm{J}, \mathrm{K}$ and $\mathrm{M}$.

- $\mathrm{Q}_{\text {int }}, \mathrm{Q}^{\ddagger}{ }_{\text {int }}$ (dimensionless) are the partition functions (center of mass factored out) of the "complex" and the transition state less external rotations, i.e., $\mathrm{Q}=\mathrm{Q}_{\text {int }} \mathrm{Q}_{\text {rot }}, \mathrm{Q}^{\ddagger}=\mathrm{Q}_{\text {int }}^{\ddagger} \mathrm{Q}_{\text {rot. }}^{\ddagger}$ For the transition state, $\mathrm{Q}^{\ddagger}$ int includes only internal degrees of freedom (including internal rotations). For the "complex", $\mathrm{Q}_{\text {int }}$ includes the relative motion of the reactants within a layer $\delta$ as well as these (now internal) rotational degrees of freedom of $\mathrm{A}$ and $\mathrm{B}$ that were not counted toward external rotations.

-The notations $\mathrm{Q}_{\text {int }+\mathrm{A}^{\prime}}(\mathrm{J}, \mathrm{T}), \mathrm{Q}^{\ddagger}{ }_{\mathrm{int}+\mathrm{A}^{\prime}}(\mathrm{J}, \mathrm{T})$ (dimensionless) are used for the "internal" partition functions + external 1D rotation about the $a$ axis (quantum number $\mathrm{K}$ ) of the "complex" and the transition state, respectively. These partition functions depend on the total angular momentum, $\mathrm{J}$, because the range of $\mathrm{K}$ is J-limited. 
- $\mathrm{Q}_{\mathrm{B}}, \mathrm{Q}_{\mathrm{B}}^{\ddagger}$ (dimensionless) are the partition functions of a $2 \mathrm{D}$ rotor associated with the quantum numbers $\mathrm{J}$ and $\mathrm{M}$ for the "complex" and the transition state, respectively.

-Similar notations are used for the densities and the numbers of states.

-The following notations are used for the equilibrium populations and the partial partition functions related to the external rotations of a prolate symmetric top with the rotational constants $\mathrm{A}>\mathrm{B}=\mathrm{C}, \mathrm{A}^{\prime}=\mathrm{A}-\mathrm{B}$. The quantum numbers, the energy spectrum and the degeneracies $\mathrm{g}$ are

$$
\begin{gathered}
J=0,1,2,3, \ldots, \infty \\
K=-J,-J+1,-J+2, \ldots,-1,0,1, \ldots, J-2, J-1, J \\
M=-J,-J+1,-J+2, \ldots,-1,0,1, \ldots, J-2, J-1, J \\
E(J, K, M)=B J(J+1)+A^{\prime} K^{2} \quad A^{\prime}=A-B \\
g(J, K, M)=1 \\
g(J, K)=\sum_{M=-J}^{J} g(J, K, M)=2 J+1
\end{gathered}
$$

The equilibrium fraction in a specific $\mathrm{J}, \mathrm{K}$ energy level is

$$
\begin{aligned}
x(J, K)=\frac{g(J, K)}{Q_{\text {rot }}} \exp (- & \left.\frac{E(J, K)}{k T}\right)=\frac{g(J, K)}{Q_{\text {rot }}} \times \\
& \exp \left(-\frac{B J(J+1)}{k T}\right) \exp \left(-\frac{A^{\prime} K^{2}}{k T}\right)
\end{aligned}
$$

The partial partition function for the K-rotor is defined as

$$
Q_{\mathrm{rot}, A^{\prime}}(J, T)=\sum_{K=-J}^{J} g(J, K) \exp \left(-\frac{A^{\prime} K^{2}}{k T}\right)
$$

Then the total partition function for the external rotations and the equilibrium fractions of J-levels are

$$
\begin{array}{r}
Q_{\mathrm{rot}}(T)=\sum_{J=0}^{\infty} Q_{\mathrm{rot}, A^{\prime}}(J, T) \exp \left(-\frac{B J(J+1)}{k T}\right) \\
x(J)=\sum_{K=-J}^{J} x(J, K)=\frac{Q_{\mathrm{rot}, A^{\prime}}(J, T)}{Q_{\mathrm{rot}}} \exp \left(-\frac{B J(J+1)}{k T}\right)
\end{array}
$$

Microcanonical Rate Constant. In the initial discussion, to illustrate the main point, we will ignore the impact of the conservation of angular momentum, of possible degrees of freedom with conserved energy, and quantum effects (tunneling and above-the-barrier reflection). The centrifugal entrance barrier as well as a possible entrance barrier (such as one caused by the zero-point vibrational energies) are ignored as well.

For reaction E1, let us consider a specific transition state (critical surface) at a fixed value of the reaction coordinate, $\mathrm{q}^{\ddagger}$, and focus on a single selected state of the transition state with the energy $E^{\prime f_{i}}$ accessible from the current energy of reactants, $\mathrm{E}$. The energies $\mathrm{E}$ and $\mathrm{E}^{\prime \neq}{ }_{\mathrm{i}}$ are measured relative to a common reference level. The difference is the energy of translational motion of the transition state along the reaction coordinate, $\mathrm{E}_{\mathrm{t}, \mathrm{i}}^{\ddagger}$ :

$$
E_{\mathrm{t}, \mathrm{i}}^{\ddagger}=E-{E^{\prime}}_{\mathrm{i}}^{\ddagger} \quad E_{\mathrm{t}, \mathrm{i}}>0
$$

On the basis of either the microcanonical equilibrium of the reactants with the "transition complex" (the transition state that is allowed to perform decoupled translational motion in a thin layer around the dividing surface $)^{28}$ or the quasi-classical derivation based on the flux of representing points in the phase space, ${ }^{30}$ it is shown that all internal states of the transition state that are accessible at a given energy (i.e., with energy below the energy of the reactants) contribute equally to the rate constant:

$$
k_{\mathrm{i}}(E)=\frac{1}{h \rho_{\mathrm{R}}(E)}
$$

The total rate constant is obtained by multiplication of E4a by the total number of accessible states of the TS:

$$
k(E)=\frac{W^{\ddagger}\left(E^{+}\right)}{h \rho_{\mathrm{R}}(E)} \quad E^{+}=E-E_{0}
$$

where $\mathrm{W}^{\ddagger}\left(\mathrm{E}^{+}\right)$is the total number of states of the transition state with the energy below $\mathrm{E}^{+}$, counting from the ground state of the transition state, $\mathrm{E}^{+}=\mathrm{E}-\mathrm{E}_{0}$. Equation $\mathrm{E} 4 \mathrm{~b}$ is the wellknown result of micro-canonical RRKM theory as applied to unimolecular reactions. ${ }^{46,47}$ as well as microcanonical TST applied to bimolecular reactions. ${ }^{30}$

Canonical Rate Constant Ignoring Restrictions Associated with Angular Momentum, Entrance Barriers and Tunneling/ Reflection. Now we incorporate the equilibrium thermal distribution E5 of the reactants to formulate the canonical expression for the rate constant.

$$
\mathrm{d} x(E)=\frac{1}{Q_{\mathrm{R}}} \exp \left(-\frac{E}{k T}\right) \rho_{\mathrm{R}}(E) \mathrm{d} E
$$

For the canonical rate constant with a positive barrier, $\mathrm{E}_{0}>0$, one derives (using $\mathrm{E}^{+}=\mathrm{E}-\mathrm{E}_{0}$, and $\mathrm{k}\left(\mathrm{E}^{+}<0\right)=0$ :

$$
\begin{aligned}
k(T)= & \frac{1}{Q_{\mathrm{R}}} \int_{E_{0}}^{\infty} \exp (-E / k T) \rho_{\mathrm{R}}(E) k\left(E^{+}\right) \mathrm{d} E= \\
& \frac{k T \exp \left(-E_{0} / k T\right)}{h Q_{\mathrm{R}}} \int_{0}^{\infty} W^{\ddagger}\left(E^{+}\right) \exp \left(-E^{+} / k T\right) \frac{\mathrm{d} E^{+}}{k T}
\end{aligned}
$$

Equation E6 as well as some further developments are equivalent to the results obtained in previous works ${ }^{18,19}$ and are given here for clarity.

Evaluation of the second integral in E6 by parts, using exp$\left(-E^{+} / k T\right) \mathrm{d} E=-k T \mathrm{~d}\left(\exp \left(-E^{+} / k T\right)\right)$ and $\mathrm{d} W^{\ddagger}\left(E^{+}\right)=\rho^{\ddagger}\left(E^{+}\right)$ $\mathrm{d} E^{+}$, results in the standard expression of transition state theory (the second integral in E6, $\int_{0}^{\infty} W^{\ddagger}\left(E^{+}\right) \exp \left(-E^{+} / k T\right) \mathrm{d} E^{+} / k T$, is the partition function of the transition state):

$$
\begin{aligned}
k(T) & =\frac{k T \exp \left(-E_{0} / k T\right)}{h \quad Q_{\mathrm{R}}} \int_{0}^{\infty} \rho^{\ddagger}\left(E^{+}\right) \exp \left(-E^{+} / k T\right) \mathrm{d} E^{+}= \\
& \frac{k T}{h} \frac{Q^{\ddagger}}{Q_{\mathrm{R}}} \exp \left(-E_{0} / k T\right) \\
& =\frac{k T}{h} \frac{Q_{\text {total }}^{\ddagger}}{Q_{\mathrm{A}} Q_{\mathrm{B}}} \exp \left(-E_{0} / k T\right)=k_{\mathrm{TST}}
\end{aligned}
$$

where $Q_{\mathrm{R}}$ and $Q^{\ddagger}$ are the partition functions of the reactants and the transition state, respectively, calculated relative to their ground states.

However, if the lowest energy at the position of the reaction bottleneck (the ground state of the transition state) lies below the ground state of the reactants, $\mathrm{E}_{0}<0$ ("negative barrier"), then the integration in E6 must start not from $\mathrm{E}_{0}$ (which is negative), but from zero: ${ }^{18,19}$ 


$$
\begin{aligned}
k(T)=\frac{1}{Q_{\mathrm{R}}} \int_{0}^{\infty} \exp (-E / k T) \rho_{\mathrm{R}}(E) k\left(E^{+}\right) \mathrm{d} E= \\
\frac{1}{h Q_{\mathrm{R}}} \int_{0}^{\infty} W^{\ddagger}\left(E-E_{0}\right) \exp (-E / k T) \mathrm{d} E
\end{aligned}
$$

Equation E8 is essentially the main point of the modified transition state theory - replacement of $\mathrm{E}-\mathrm{E}_{0}$ results in a positive lower integration limit, $-\mathrm{E}_{0}$, and the integral cannot be reduced to the partition function of the transition state anymore:

$$
\begin{aligned}
k(T)= & \frac{1}{h Q_{\mathrm{R}}} \int_{0}^{\infty} W^{\ddagger}\left(E-E_{0}\right) \exp (-E / k T) \mathrm{d} E= \\
& \frac{k T \exp \left(-E_{0} / k T\right)}{h Q_{\mathrm{R}}} \int_{-E_{0}}^{\infty} W^{\ddagger}\left(E^{+}\right) \exp \left(-E^{+} / k T\right) \frac{\mathrm{d} E^{+}}{k T}
\end{aligned}
$$

When the lower limit of integration is zero, the second integral in E9, $\int_{-E_{0}}^{\infty} W^{\ddagger}\left(E^{+}\right) \exp \left(-E^{+} / k T\right)\left(\mathrm{d} E^{+} / k t\right)$ is simply the partition function of the transition state. However, for negative barriers, the lower limit in this integral is positive which reduces the absolute value of the rate constant compared with the rate constant obtained by formal substitution of a negative barrier into the classical TST expression:

$$
\int_{-E_{0}}^{\infty} W^{\ddagger}\left(E^{+}\right) \exp \left(-E^{+} / k T\right) \frac{\mathrm{d} E^{+}}{k T}<Q^{\ddagger} \quad-E_{0}>0
$$

Moreover, this has an impact on the temperature dependence, again, compared with the result obtained by formal substitution of negative values for the barrier into the classical TST. The negative temperature dependence predicted by E9 is weaker than one obtained by classical TST with negative $\mathrm{E}_{0}$.

It is instructive to analyze the temperature dependence predicted by equation E9 in an ultimate case of large "negative barriers", $-\mathrm{E}_{0} \gg \mathrm{kT}$. Integrating E8 by parts, and expanding the density of states of the transition state into the Taylor series near $-\mathrm{E}_{0}$, one obtains

$$
\begin{aligned}
\int_{0}^{\infty} W^{\ddagger}\left(E-E_{0}\right) \exp (-E / k T) \frac{\mathrm{d} E}{k T} & = \\
& W^{\ddagger}\left(-E_{0}\right)+k T \rho^{\ddagger}\left(-E_{0}\right)+\left.(k T)^{2} \frac{\mathrm{d} \rho^{\ddagger}}{\mathrm{d} E}\right|_{\left(-E_{0}\right)}+\ldots
\end{aligned}
$$

Neglecting all terms in E11 but the first one results in the ultimate expression for the rate constant for reactions with large negative barriers:

$$
k(T)=\frac{k T}{h} \frac{W^{\ddagger}\left(-E_{0}\right)}{Q_{\mathrm{R}}}
$$

It should be stressed that the center-of-mass motion is factored out in the partition function of the reactants, $Q_{R}$, but not the relative motion of the reactants. The partition function $Q_{R}$ includes electronic, vibrational, and rotational degrees of freedom as well as the relative translational motion of the reactants. E12:

There are several conclusions that can be derived from Eq.

(i) For similar negative barriers, the more complex the reactants, the larger is the absolute value of the negative temperature dependence of the rate constant.

(ii) Because the number of states in the transition state increases faster than the partition function with the complexity of the reactants, in a series of similar reactions, reactions of more complex reactants are expected to be faster.

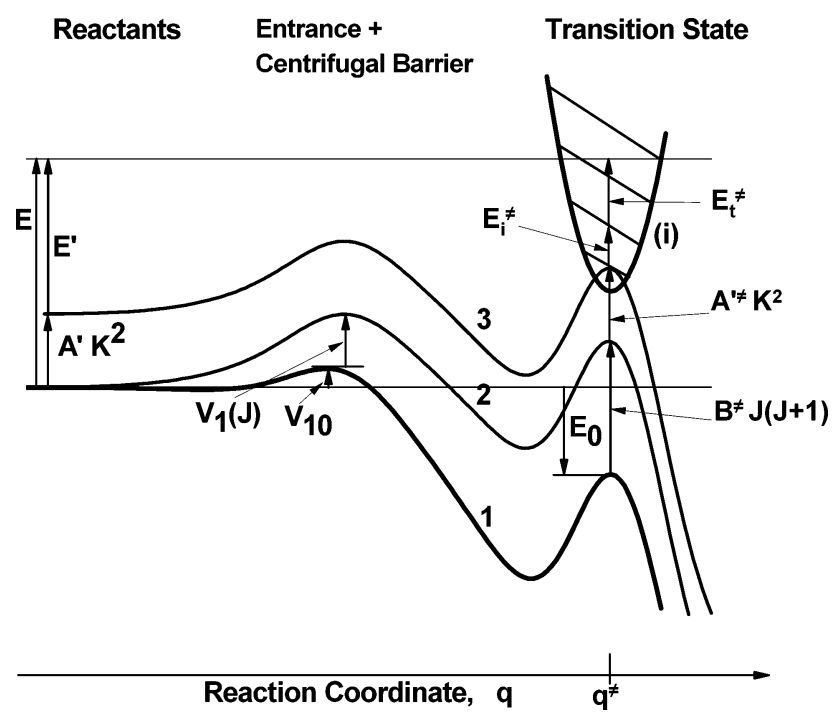

Figure 1. Sketch of the energy diagram. Curve 1: adiabatic energy $+E_{z}$ (the ground state energy of the system). Curve 2: the $J$ contribution of the external rotation is added (adiabatic degree of freedom). Curve 3: the $K$-contribution of the external rotation is added (assumed to be conserved).

(iii) All neglected terms in E11 have positive temperature dependences; therefore the actual negative temperature dependence is weaker than that predicted by Eq. E12.

(iv) All neglected terms in E11 are positive; therefore eq E12 represents a lower limit on the rate constant (within the assumptions of the theory).

Conclusions i-iii are in good qualitative agreement with the experimental observations. ${ }^{1,2,11-13}$ However, for quantitative calculations, conservation of angular momentum, the role of the K quantum number, the role of a possible additional barrier at large separations of the reactants (such as due to zero-point vibrational energies or an inherent saddle point in the PES), the entrance centrifugal barrier and tunneling/above-the-barrier reflection should be taken in consideration.

Taking into Account Conservation of Angular Momentum $(\boldsymbol{J}$ - Adiabatic), Conservation of Energy of $\boldsymbol{K}$-Rotor (KRotor Is Nonactive), and Quantum Corrections (Tunneling + Above-the-Barrier Reflection). The derivation will be performed initially ignoring a possible entrance barrier as well as the centrifugal barriers. These will be taken into account at a later stage.

Figure 1 illustrates the energy diagram and the notations used in the derivation. We assume that K-rotation (the rotation about the $a$-axis) is a degree of freedom with conserved energy. It can be argued ${ }^{16}$ that for the reaction $\mathrm{CH}_{3}+\mathrm{HBr}$ the K-rotor should be treated as a degree of freedom with fixed energy (i.e., nonactive degree of freedom). This is based on the fact that $A^{\ddagger}$ $\gg \mathrm{B}^{\ddagger}$, and that $\mathrm{A}$ does not change appreciably in the course of reaction. For the reaction $\mathrm{CH}_{3}+\mathrm{HBr}$, the rotational constant $\mathrm{A}^{\ddagger}$ is much larger than the rotational constant $\mathrm{B}^{\ddagger}$ (ca. 37 times). In addition, this rotational constant changes negligibly when going from the reactants $(A=142.5 \mathrm{GHz})$ to the transition state $\left(A^{\ddagger}=145.3 \mathrm{GHz}\right)$. Therefore, $\mathrm{B}^{\ddagger}$ could be neglected in the energy term associated with quantum number $\mathrm{K}$. Because $\mathrm{K}$ is a good quantum number for a symmetric top, the K-contribution of the rotational energy is conserved with good accuracy.

Because the distribution function for translational motion along the reaction coordinate of the transition states that are in internal states which lie below the ground state of the reactants is not Maxwell's distribution, but a truncated Maxwell's 
distribution, the usual expressions for the canonical transmission probability are not applicable. To incorporate quantum mechanical tunneling and above-the-barrier reflection, the microcanonical transmission probability should be used. We use the one-dimensional transmission probability function given in ref 46:

$$
p\left(E_{\mathrm{t}}^{\ddagger}\right)=\frac{1}{1+\exp \left(-2 \pi E_{\mathrm{t}}^{\ddagger} / h v^{*}\right)}
$$

where $E_{\mathrm{t}}^{\ddagger}$ is the translational energy of the TS for the motion along the reaction coordinate, and $v^{*}$ is the absolute value of the imaginary frequency of the TS. The transmission function $p\left(E_{\mathrm{t}}^{\ddagger}\right)$ replaces the Heaviside stepwise transmission function which is used in the classical description. The characteristic energy width of the transmission function $\mathrm{E} 13$ is $\pm h v^{*} / 2 \pi$. This is ca. $80 \mathrm{~cm}^{-1}$, or $1 \mathrm{~kJ} \mathrm{~mol}^{-1}$, for the $\mathrm{H}_{3} \mathrm{C}-\mathrm{H}-\mathrm{Br}$ transition state.

In the derivation below the notion of an artificial complex $\mathrm{C}$ is invoked (see Appendix 1). The contribution of a specific internal level $\mathrm{i}$ of the transition state to the $J, K$-specific microcanonical rate constant of the complex transformation into the products is (where $\mathrm{E}=\mathrm{E}^{\prime}+\mathrm{E}_{\mathrm{A}}(\mathrm{K})$ is the total energy of the reactants less the energy of the center of mass motion):

$$
k_{C, J, K, i}(E)=\frac{p\left(E-E_{0}-E_{i}^{\ddagger}-E_{\mathrm{B}}^{\ddagger}(J)-E_{\mathrm{A}}^{\ddagger}(K)\right)}{h \rho_{\mathrm{int}}\left(E-E_{\mathrm{A}}(K)\right)}
$$

and the difference $E-E_{0}-E_{i}^{\ddagger}-E_{\mathrm{B}}^{\ddagger}(J)-E_{\mathrm{A}}^{\ddagger}(K)=E_{\mathrm{t}}^{\neq}$is the energy of translational motion along the reaction coordinate. The transmission probability function $\mathrm{p}\left(E_{\mathrm{t}}^{\ddagger}\right)$ has nonzero values for negative translational energies (tunneling) and it is not equal to one at positive energies (above-the-barrier reflection). To obtain the canonical $i, J, K$-specific rate constant, E14 is averaged with the equilibrium distribution function of the reactants, using $E^{\prime}=E-E_{\mathrm{A}}(K)$, and the assumption that the $K$ energy is conserved, $E_{\mathrm{A}}^{\ddagger}(K)=E_{\mathrm{A}}(K)$ :

$$
\begin{aligned}
k_{C, J, K, i}(T) & = \\
& \int_{0}^{\infty} \frac{p\left(E^{\prime}-E_{0}-E_{i}^{\ddagger}-E_{\mathrm{B}}^{\ddagger}(J)\right)}{h \rho_{\mathrm{int}}\left(E^{\prime}\right)} \frac{\rho_{\mathrm{int}}\left(E^{\prime}\right) \mathrm{d} E^{\prime}}{Q_{\mathrm{int}}} \exp \left(-E^{\prime} / k T\right) \\
& =\frac{1}{h Q_{\mathrm{int}}} \int_{0}^{\infty} p\left(E^{\prime}-E_{0}-E_{i}^{\ddagger}-E_{\mathrm{B}}^{\ddagger}(J)\right) \exp \left(-E^{\prime} / k T\right) \mathrm{d} E^{\prime}
\end{aligned}
$$

When $E^{\prime}-E_{0}-E_{i}^{\ddagger}-E_{\mathrm{B}}^{\ddagger}(J)$ is replaced with the translational energy, E15 becomes

$$
\begin{aligned}
& k_{C, J, K, i}(T)= \\
& \quad \frac{k T}{h Q_{\text {int }}} \exp \left(-E_{i}^{\ddagger}(J) / k T\right) \int_{-E_{i}^{\ddagger}(J)}^{\infty} \exp \left(-E_{\mathrm{t}}^{\ddagger} / k T\right) p\left(E_{\mathrm{t}}^{\ddagger}\right) \mathrm{d} E_{\mathrm{t}}^{\ddagger} / k T
\end{aligned}
$$

where

$$
E_{i}^{\ddagger}(J)=E_{0}+E_{i}^{\ddagger}+E_{\mathrm{B}}^{\ddagger}(J)
$$

For the internal states with large positive energies, e.g., $E_{i}^{\ddagger}(J)$ $>3 h v^{*} / 2 \pi$, the lower integration limit can be replaced by $-\infty$, and a universal canonical transmission coefficient appears as a common factor: ${ }^{45,48}$

$\begin{aligned} & \chi(T)=\int_{-\infty}^{\infty} \exp \left(-E_{\mathrm{t}} / k T\right) p\left(E_{\mathrm{t}}\right) \mathrm{d} E_{\mathrm{t}} / k T=\frac{h v^{*} / 2 k T}{\sin \left(h v^{*} / 2 k T\right)} \\ & \text { for } \quad h v^{*} / 2 k T<\pi(\mathrm{E} 18)\end{aligned}$

The total canonical rate constant is obtained by summation of E16 over the distribution function of the reactants and the internal states of the transition state:

$$
\begin{aligned}
k_{\mathrm{C}}(T) & =\frac{k T}{h Q_{\text {int }}} \exp \left(-\frac{E_{0}}{k T}\right) \times \\
& \sum_{i=0}^{\infty} \exp \left(-\frac{E_{i}^{\ddagger}}{k T}\right) \sum_{J=0}^{\infty} \sum_{K=-J}^{J} \frac{1}{Q_{\text {rot }}} g(J, K) \exp \left(-\frac{B^{\ddagger} J(J+1)}{k T}\right) \times \\
& \exp \left(-\frac{A^{\prime} K^{2}}{k T}\right) \int_{-E_{0}-E_{i}^{\ddagger}-B^{\ddagger} J(J+1)}^{\infty} \exp \left(-\frac{E_{\mathrm{t}}^{\ddagger}}{k T}\right) p\left(E_{\mathrm{t}}^{\ddagger}\right) \frac{\mathrm{d} E_{\mathrm{t}}^{\ddagger}}{k T}(\mathrm{E}
\end{aligned}
$$

Note that $E_{i}^{\ddagger}(J)$ were calculated relative to the ground state of the reactants, whereas $E_{i}^{\neq}$are the energies of the internal state of the transition state relative to its ground state. For large positive barriers the lower integration limit in the integral E19 can be replaced by $-\infty$, the integral becomes the canonical transmission coefficient $\chi(T)$ (E18), and the whole expression E19 is the expression for the rate constant of the classical transition state theory corrected for quantum effects. Here $A^{\ddagger}$ $=A$ and conversion of the "complex" reaction rate constant to the bimolecular rate constant (Appendix 1) are used:

$$
\begin{aligned}
& k_{\mathrm{C}}=\chi(T) \frac{k T}{h Q_{\text {int }} Q_{\text {rot }}} \exp \left(-\frac{E_{0}}{k T}\right) \times \\
& \sum_{i=1}^{\infty} \exp \left(-\frac{E_{i}^{\ddagger}}{k T}\right) \sum_{J=0}^{\infty} \sum_{K=-J}^{J} g(J, K) \exp \left(-\frac{B^{\ddagger} J(J+1)}{k T}\right) \exp \left(-\frac{A^{\ddagger} K^{2}}{k T}\right) \\
& =\chi(T) \frac{k T Q_{\text {int }}^{\ddagger} Q_{\text {rot }}^{\ddagger}}{h Q_{\text {int }} Q_{\text {rot }}} \exp \left(-\frac{E_{0}}{k T}\right)=\chi(T) \frac{k T Q^{\ddagger}}{h Q} \exp \left(-\frac{E_{0}}{k T}\right)= \\
& \chi(T) \frac{k T Q_{\text {total }}^{\ddagger}}{h Q_{\mathrm{C}}} \exp \left(-\frac{E_{0}}{k T}\right) \\
& k=\frac{Q_{\mathrm{C}}}{Q_{\mathrm{A}} Q_{\mathrm{B}}} k_{\mathrm{C}}=\frac{Q_{\mathrm{C}}}{Q_{\mathrm{A}} Q_{\mathrm{B}}} \chi(T) \frac{k T Q_{\text {total }}^{\ddagger}}{h Q_{\mathrm{C}}} \exp \left(-\frac{E_{0}}{k T}\right)= \\
& \chi(T) \frac{k T Q_{\text {total }}^{\ddagger}}{h Q_{\mathrm{A}} Q_{\mathrm{B}}} \exp \left(-\frac{E_{0}}{k T}\right)=k_{\mathrm{TST}}
\end{aligned}
$$

Therefore, it is convenient to complete the integral in E19 by extending the lower limit of integration to $-\infty$, and to express the difference as a correction:

$$
\begin{gathered}
k(T)=k_{\mathrm{TST}}(1-\delta) \\
\delta=\sum_{i=0}^{\infty} \delta_{i}
\end{gathered}
$$




$$
\begin{gathered}
\delta_{i}=\frac{1}{Q_{\mathrm{int}}^{\ddagger} Q_{\mathrm{rot}}^{\ddagger} \chi(T)} \exp \left(-\frac{E_{i}^{\ddagger}}{k T}\right) \times \\
\sum_{J=0}^{\infty} \exp \left(-\frac{B^{\ddagger} J(J+1)}{k T}\right) \int_{-\infty}^{-E_{0}-E_{i}^{\ddagger}-B^{\ddagger} J(J+1)} \exp \left(-\frac{E_{\mathrm{t}}^{\ddagger}}{k T}\right) \times \\
=\frac{1}{p\left(E_{\mathrm{t}}^{\ddagger}\right) \frac{\mathrm{d} E_{\mathrm{t}}^{\ddagger}}{k T} \sum_{K=-J}^{J} g(J, K) \exp \left(-\frac{A^{\prime \neq} K^{2}}{k T}\right)} Q_{\mathrm{int}}^{\ddagger} Q_{\mathrm{rot}}^{\ddagger} \chi(T) \exp \left(-\frac{E_{i}^{\ddagger}}{k T}\right) \sum_{J=0}^{\infty} Q_{\mathrm{A}}^{\ddagger}(J, T) \times \\
\exp \left(-\frac{B^{\ddagger} J(J+1)}{k T}\right) \times \\
\int_{-\infty}^{-E_{0}-E_{i}^{\ddagger}-B^{\ddagger} J(J+1)} \exp \left(-\frac{E_{\mathrm{t}}^{\ddagger}}{k T}\right) p\left(E_{\mathrm{t}}^{\ddagger}\right) \frac{\mathrm{d} E_{\mathrm{t}}^{\ddagger}}{k T}(\mathrm{E} 23)
\end{gathered}
$$

Because the rotational constant $B^{\ddagger}$ is quite small (ca. $0.1 \mathrm{~cm}^{-1}$ $\ll k T \approx 200 \mathrm{~cm}^{-1}$ ), the summations in E23 are replaced with integrations.

Although the total energy is factorized in $J$ and $K$, there is still coupling between these two degrees of freedom due to the limit on the possible values of $K$ imposed by $J,|K|<J$. This is the reason the partition function of the $K$-rotor is $J$-dependent. For rotors with the rotational constant $A$ much larger than the rotational constant $B$ (which is the case for the transition state of the $\mathrm{CH}_{3}+\mathrm{HBr}$ reaction) a useful approximation is to extend the summation range in the definition of $Q_{A}^{\ddagger}(J, T)$ to infinity:

$$
\begin{aligned}
& Q_{\mathrm{rot}, \mathrm{A}}(J, T)=\sum_{K=-J}^{J} g(J, K) \exp \left(-\frac{A^{\prime} K^{2}}{k T}\right) \approx \sum_{K=-\infty}^{\infty} g(J, K) \times \\
& \exp \left(-\frac{A^{\prime} K^{2}}{k T}\right) \approx \int_{-\infty}^{\infty} g(J, K) \exp \left(-\frac{A^{\prime} K^{2}}{k T}\right) \\
&=(2 J+1) \sqrt{\pi} \sqrt{\frac{k T}{A^{\prime}}}=(2 J+1) Q_{\mathrm{rot}}^{(1)}
\end{aligned}
$$

Under this approximation E23 simplifies as

$$
\begin{aligned}
& \delta_{i}=\frac{1}{Q_{\mathrm{int}}^{\ddagger} Q_{\mathrm{B}}^{\ddagger} \chi(T)} \exp \left(-\frac{E_{i}^{\ddagger}}{k T}\right) \times \\
& \int_{0}^{\infty} \exp \left(-\frac{E_{\mathrm{B}}^{\ddagger}}{k T}\right) \frac{\mathrm{d} E_{\mathrm{B}}^{\ddagger}}{B^{\ddagger}} \int_{-\infty}^{-E_{0}-E_{\mathrm{t}}^{\ddagger}-E_{\mathrm{B}}^{\ddagger}} \exp \left(-\frac{E_{\mathrm{t}}^{\ddagger}}{k T}\right) p\left(E_{\mathrm{t}}^{\ddagger}\right) \frac{\mathrm{d} E_{\mathrm{t}}^{\ddagger}}{k T}
\end{aligned}
$$

Expression E25 can be evaluated by changing the order of integration:

$\int_{0}^{\infty} \mathrm{d} E_{\mathrm{B}}^{\ddagger} \int_{-\infty}^{-E_{0}-E_{i}^{\ddagger}-E_{\mathrm{B}}^{\ddagger}} \mathrm{d} E_{\mathrm{t}}^{\ddagger} \rightarrow \int_{-\infty}^{-E_{0}-E_{i}^{\ddagger}} \mathrm{d} E_{\mathrm{t}}^{\ddagger} \int_{0}^{-E_{0}-E_{i}^{\ddagger}-E_{\mathrm{t}}^{\ddagger}} \mathrm{d} E_{\mathrm{B}}^{\ddagger} \quad(\mathrm{E} 26)$

Therefore, (E25) becomes

$$
\begin{aligned}
\delta_{i}= & \frac{1}{Q_{\mathrm{int}}^{\ddagger} Q_{\mathrm{B}}^{\ddagger} \chi(T)} \exp \left(-\frac{E_{i}^{\ddagger}}{k T}\right) \times \\
& \int_{-\infty}^{-E_{0}-E_{i}^{\ddagger}} \exp \left(-\frac{E_{\mathrm{t}}^{\ddagger}}{k T}\right) p\left(E_{\mathrm{t}}^{\ddagger}\right) \frac{\mathrm{d} E_{\mathrm{t}}^{\ddagger}}{k T} \int_{0}^{-E_{0}-E_{i}^{\ddagger}-E_{\mathrm{t}}^{\ddagger}} \exp \left(-\frac{E_{\mathrm{B}}^{\ddagger}}{k T}\right) \frac{\mathrm{d} E_{\mathrm{B}}^{\ddagger}}{B^{\ddagger}} \\
= & \frac{1}{Q_{\mathrm{int}}^{\ddagger} Q_{\mathrm{B}}^{\ddagger} \chi(T)} \exp \left(-\frac{E_{i}^{\ddagger}}{k T}\right)\left(\frac{k T}{B^{\ddagger}}\right) \times \\
& \int_{-\infty}^{-E_{0}-E_{E^{\ddagger}}^{\ddagger}} \exp \left(-\frac{E_{\mathrm{t}}^{\ddagger}}{k T}\right) p\left(E_{\mathrm{t}}^{\ddagger}\right) \frac{\mathrm{d} E_{\mathrm{t}}^{\ddagger}}{k T}\left(1-\exp \left(\frac{E_{0}+E_{i}^{\ddagger}+E_{\mathrm{t}}^{\ddagger}}{k T}\right)\right)
\end{aligned}
$$

Because $Q_{B}^{\ddagger}=k T / B^{\ddagger}$, these two entries cancel out in (E27).

When a dimensionless energy $y_{i}=\left(-E_{0}-E_{i}^{\ddagger}\right) / k T$ is introduced, (E27) can be rewritten as

$$
\begin{aligned}
\delta_{i}=\frac{1}{Q_{\text {in }}^{\ddagger} \chi(T)} \exp \left(-\frac{E_{i}^{\ddagger}}{k T}\right) \times \\
\quad \int_{-\infty}^{y_{i}} \exp (-\xi) \tilde{p}(a \xi) \mathrm{d} \xi\left(1-\exp \left(-\left(y_{i}-\xi\right)\right)\right) \quad(\mathrm{E} 28)
\end{aligned}
$$

where the function $\tilde{p}(x)=1 /(1+\exp (-x))$, and $a=2 \pi k T /$ $h v^{*}$. When the partial transmission coefficient, $\chi(y, a)$,

$$
\chi(y, a)=\int_{-\infty}^{y} \exp (-\xi) \tilde{p}(a \xi) \mathrm{d} \xi=\int_{-\infty}^{y} \frac{\exp (-\xi)}{1+\exp (-a \xi)} \mathrm{d} \xi
$$

is introduced, (E28) becomes

$$
\begin{aligned}
\delta_{i}= & \frac{1}{Q_{\text {int }}^{\ddagger} \chi(T)} \times \\
& \exp \left(-\frac{E_{i}^{\ddagger}}{k T}\right)\left\{\chi\left(y_{i}, a\right)-\exp \left(-y_{i}\right) \frac{1}{a} \ln \left(1+\exp \left(a y_{i}\right)\right)\right\}
\end{aligned}
$$

Taking into Account a Possible Entrance Barrier and the Centrifugal Barriers. Because centrifugal energy decays as $1 / r^{2}$ with the separation of the reactants, and the "potential energy" asymptotically decays much faster, as about $1 / r^{6}$, a centrifugal barrier appears at large separations of the reactants. The heights $\left(V_{1}(J)\right)$ and the positions of the centrifugal barriers depend on $J$. Because these centrifugal barriers are quite wide (the corresponding imaginary frequencies are small), the quantum effects (tunneling and above-the-barrier reflection) are negligible. ${ }^{46}$ It should be stressed that these barriers do not represent another transition state but serve just as energy cutoff barries; that is, no reflection of the trajectories with energies above the barriers at the barrier positions is assumed. To take the entrance barrier into account, the lower integration limit in E15 now should be replaced by $V_{1}(J)$ :

$$
k_{C, J, K, i}(T)=\int_{V_{1}(J)}^{\infty} \frac{p\left(E^{\prime}-E_{0}-E_{i}^{\ddagger}-E_{\mathrm{B}}^{\ddagger}(J)\right)}{h \rho_{\text {int }}\left(E^{\prime}\right)} \frac{\rho_{\text {int }}\left(E^{\prime}\right) \mathrm{d} E^{\prime}}{\begin{array}{c}
Q_{\text {int }} \\
\exp \left(-E^{\prime} / k T\right)
\end{array}}
$$

A series of transformations similar to those presented in the previous section leads to the corrections $\delta_{i}$ : 


$$
\begin{aligned}
\delta_{i}= & \frac{1}{Q_{\mathrm{int}}^{\ddagger} Q_{\mathrm{B}}^{\ddagger} \chi(T)} \exp \left(-\frac{E_{i}^{\ddagger}}{k T}\right) \times \\
& \int_{0}^{\infty} \exp \left(-\frac{E_{\mathrm{B}}^{\ddagger}}{k T}\right) \frac{\mathrm{d} E_{\mathrm{B}}^{\ddagger}}{B^{\ddagger}} \int_{-\infty}^{V_{1}(J)-E_{0}-E_{i}^{\ddagger}-E_{\mathrm{B}}^{\ddagger}(J)} \exp \left(-\frac{E_{\mathrm{t}}^{\ddagger}}{k T}\right) p\left(E_{\mathrm{t}}^{\ddagger}\right) \frac{\mathrm{d} E_{\mathrm{t}}^{\ddagger}}{k T}
\end{aligned}
$$

Now, another useful approximation can be made if there is an additional barrier in the entrance of the reactant valley, $V_{10}$, such as one caused by the zero-point vibrational energy. The position of this barrier will approximately fix the positions of the centrifugal barriers, so that an approximately $J$-independent rotational constant that corresponds to the barrier geometry, $B_{1}$, can be introduced. Then

$$
V_{1}(J)=V_{10}+B_{1} J(J+1)
$$

Because $E_{\mathrm{B}}^{\ddagger}=B^{\ddagger} J(J+1)$, the entrance barrier $V_{10}$ is combined with the negative barrier $E_{0}$, and the entrance centrifugal energy is combined with the centrifugal energy of the transition state:

$$
\begin{gathered}
E_{0}^{\prime}=E_{0}-V_{10} \\
E_{\mathrm{B}}^{\ddagger}(J)-V_{1}(J)=E_{\mathrm{B}}^{\ddagger}(J) /(1+b)
\end{gathered}
$$

where

$$
b=\frac{B_{1}}{B^{\ddagger}-B_{1}}
$$

Introducing dimensionless variables $\left(E_{\mathrm{B}}^{\ddagger} / k T=x, E_{\mathrm{t}}^{\ddagger} / k T=\xi\right)$ and changing the order of integration, the integral in E32 is reduced to

$$
\begin{aligned}
I & =\int_{0}^{\infty} \exp \left(-\frac{E_{\mathrm{B}}^{\ddagger}}{k T}\right) \frac{\mathrm{d} E_{\mathrm{B}}^{\ddagger}}{B^{\ddagger}} \times \\
& =Q_{\mathrm{B}}^{\ddagger} \int_{0}^{V_{1}(J)-E_{0}-E_{i}^{\ddagger}-E_{\mathrm{B}}^{\ddagger}(J)} \exp \left(-\frac{E_{\mathrm{t}}^{\ddagger}}{k T}\right) p\left(E_{\mathrm{t}}^{\ddagger}\right) \frac{\mathrm{d} E_{\mathrm{t}}^{\ddagger}}{k T} \\
& =Q_{\mathrm{B}}^{\ddagger} \int_{-\infty}^{y_{i}} \exp (-\xi) \mathrm{d} x \int_{-\infty}^{y_{i}^{\prime}-x /(b+1)} \exp (-\xi) p(a \xi) \mathrm{d} \xi= \\
& Q_{\mathrm{B}}^{\ddagger} \int_{-\infty}^{y_{i}^{\prime}} \exp (-\xi) p(a \xi) \mathrm{d} \xi \int_{0}^{(b+1)\left(y_{i}-\xi\right)} \exp (-x) \mathrm{d} x \\
& =Q_{\mathrm{B}}^{\ddagger}\left\{\chi \left(1-\exp \left(-(b+1) y_{i}\right) \times\right.\right. \\
& \left.\exp ((b)-\exp (-(b+1) \xi)) y_{-\infty}^{y_{i}} \exp (b \xi) p(a \xi) \mathrm{d} \xi\right\}
\end{aligned}
$$

The integral in the last term can be defined as a "generalized transmission function", $\operatorname{Tr}(a, b, x)$, and evaluated via a hypergeometric function ${ }_{2} F_{1}$ (see Appendix 2):

$$
\begin{aligned}
& \operatorname{Tr}(a, b, x)=\int_{-\infty}^{x} \frac{\exp (b \xi)}{1+\exp (-a \xi)} \mathrm{d} \xi= \\
& \frac{\exp (b x)(1+\exp (a x))}{(a+b)(1+\exp (-a x))^{2}} F_{1}\left(1,(1+b / a),(2+b / a),\left(-\mathrm{e}^{a x}\right)\right)
\end{aligned}
$$

Evaluation of the generalized transmission function is discussed in Appendix 2.
Summary. Modified TST for Reactions with "Negative Barriers". Taking into Account Conservation of Angular Momentum, the Entrance Barrier, the Entrance Centrifugal Barriers and Tunneling + Reflection. Rotation $K$ Treated as a "Fixed Energy" Degree of Freedom. Under the assumptions stated in the title, the expressions of the modified transition theory for reactions with negative barriers are as follows.

- The reaction bottleneck has a negative energy relative to the ground state of the reactants, $E_{0}=-\left|E_{0}\right|<0$. For high enough positive barriers $\left(>5 \mathrm{~kJ} \mathrm{~mol}^{-1}\right)$, the correction $\delta$ vanishes, and the rate constant is given by the transition state theory.

$$
k_{\mathrm{TST}}=\chi(T) \frac{k T}{h} \frac{Q_{\mathrm{total}}^{\ddagger}}{Q_{\mathrm{A}} Q_{\mathrm{B}}} \exp \left(-\frac{E_{0}}{k T}\right)
$$

(the partition functions $Q_{\mathrm{A}}, Q_{\mathrm{B}}$ and $Q_{\text {total }}^{\ddagger}$ are the total partition functions including translational motions of the center of mass).

- $\quad k=k_{\mathrm{MTST}}=k_{\mathrm{TST}}(1-\delta)$

(the rate constant of the Modified Transition State Theory)

$$
\text { - } \quad \delta=\delta_{0}+\delta_{1}+\delta_{2}+\ldots+\delta_{i}+\ldots
$$

(formally the summation is extended to infinity; in practice, the series can be truncated for the internal states of the TS exceeding $\left|\mathrm{E}_{0}\right|$ by several $h v^{*} / 2 \pi$, i.e., by ca. $400 \mathrm{~cm}^{-1} \approx 5 \mathrm{~kJ} \mathrm{~mol}^{-1}$ for the title reaction).

$$
\begin{gathered}
-\delta_{i}=\frac{1}{Q_{\mathrm{int}}^{\ddagger} \chi(T)} \exp \left(-E_{i}^{\ddagger} / k T\right) H\left(a, b, y_{i}\right) \\
y_{i}=\frac{V_{01}-E_{0}-E_{i}^{\ddagger}}{k T} \\
a=\frac{2 \pi k T}{h v^{*}} \\
b=\frac{B_{1}}{B^{\ddagger}-B_{1}}
\end{gathered}
$$

- $H\left(a, b, y_{i}\right)=\operatorname{Tr}\left(a,-1, y_{i}\right)-\exp \left(-(1+b) y_{i}\right) \operatorname{Tr}\left(a, b, y_{i}\right)$

$$
\begin{aligned}
& -\operatorname{Tr}(a, b, x)=\int_{-\infty}^{x} \frac{\exp (b \xi)}{1+\exp (-a \xi)} \mathrm{d} \xi= \\
& \frac{\exp (b x)(1+\exp (a x))}{(a+b)(1+\exp (-a x))^{2}} F_{1}\left(1,(1+b / a),(2+b / a),\left(-\mathrm{e}^{a x}\right)\right)
\end{aligned}
$$

$$
\chi(T)=\operatorname{Tr}(a,-1, \infty)=\frac{(\pi / a)}{\sin (\pi / a)}
$$

The set of equations E39 includes all the expressions required for the evaluation of the rate constant $k_{\text {MTST }}$. Evaluation of the hypergeometric function ${ }_{2} F_{1}$ is outlined in Appendix 2 .

\section{Results and Discussion}

Theoretical Calculations of the Potential Energy Surface. Stationary points and the reaction path for reaction 1 were investigated using spin-unrestricted QCISD theory with the allelectron $6-311 \mathrm{G}(\mathrm{d}, \mathrm{p})$ atomic basis set. The reactants, a loosely bound complex and a transition state (TS) between this species 
TABLE 1: Structures, Frequencies and Energies of Stationary Points on the $\mathbf{C H}_{\mathbf{3}}+\mathbf{H B r}$ Potential Energy Surface

\begin{tabular}{|c|c|c|c|c|c|c|c|c|c|}
\hline species & $R(\mathrm{C}-\mathrm{H})^{a}$ & $R\left(\mathrm{C}-\mathrm{H}^{\prime}\right)^{a}$ & $\angle \mathrm{HCH}^{\prime} a$ & $R\left(\mathrm{H}^{\prime}-\mathrm{Br}\right)^{a}$ & frequencies $^{a}$ & $\operatorname{CCSD}(\mathrm{T}) / \mathrm{DZ}^{b}$ & $\operatorname{CCSD}(\mathrm{T}) / \mathrm{TZ}^{b}$ & $\operatorname{CCSD}(\mathrm{T}) / \mathrm{QZ}^{b}$ & $\operatorname{CCSD}(\mathrm{T}) / \mathrm{CBS}^{b}$ \\
\hline $\mathrm{CH}_{3}$ & 1.083 & $\infty$ & 90.0 & & $415,1378(2), 3002,3176(2)$ & -39.716038 & -39.760920 & -39.772372 & -39.776295 \\
\hline & & & & 1.416 & 2581 & -41 & -41 & -41 & 692 \\
\hline & 1.084 & 2.529 & 92.7 & & $\begin{array}{l}\text { 63, 92(2), 219(2), 519, 1379(2), } \\
\quad 2531,2996,3171(2)\end{array}$ & -455.935933 & -456.056743 & -456.089190 & -456.101105 \\
\hline TS & 1.086 & 1.613 & 99.2 & 1.509 & $\begin{array}{l}497 \mathrm{i}, 287(2), 683,687(2), 1236 \\
1385(2), 2984,3146(2)\end{array}$ & -455.933589 & -456.055584 & -456.088519 & -456.100698 \\
\hline $\begin{array}{l}\mathrm{CH}_{4} \\
\mathrm{Br}\end{array}$ & 1.093 & 1.093 & 109.5 & $\infty$ & $1312(3), 1510(2), 2923,3038(3)$ & $\begin{array}{r}-40.387346 \\
-415.581088\end{array}$ & $\begin{array}{r}-40.438054 \\
-415.649270\end{array}$ & $\begin{array}{r}-40.450817 \\
-415.667168\end{array}$ & $\begin{array}{r}-40.455109 \\
-415.673538\end{array}$ \\
\hline
\end{tabular}

${ }^{a}$ QCISD/6-311G(d,p) data. Bond lengths in $10^{-10} \mathrm{~m}$, angles in degrees and scaled vibrational frequencies in $\mathrm{cm}^{-1}$. ${ }^{b}$ Single-point coupled cluster calculations using cc-pVnZ-PP basis sets with $n=2-4$, and extrapolation to the complete basis set limit, in au $\left(1 \mathrm{au} \approx 2625.5 \mathrm{~kJ}^{\mathrm{mol}}{ }^{-1}\right)$.

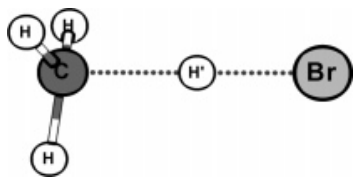

Figure 2. Sketch of the $\mathrm{CH}_{3}+\mathrm{HBr}$ orientation along the $C_{3 v}$ reaction coordinate. See Table 1 for geometry parameters.

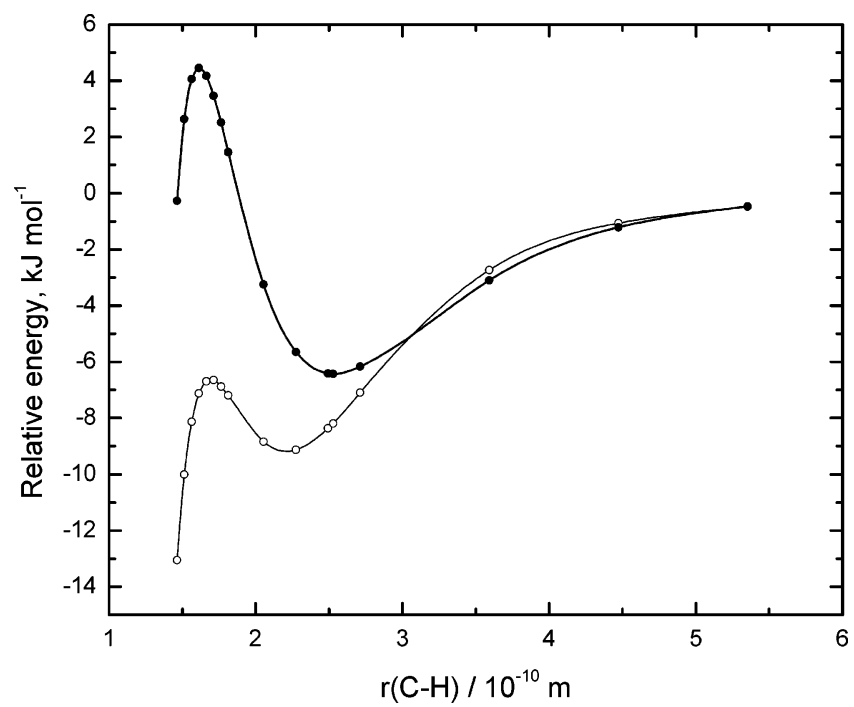

Figure 3. Energy profile for reaction 1a (no zero-point vibrational energy included) along the QCISD/6-311G(d,p) reaction coordinate, shown as a function of the length of the forming $\mathrm{C}-\mathrm{H}$ bond, for two levels of theory. The upper curve is QCISD/6-311G(d,p), and the lower is $\operatorname{CCSD}(\mathrm{T}) / \mathrm{CBS}$.

and the final products $\mathrm{CH}_{4}+\mathrm{Br}$ were characterized, and their geometries and frequencies are summarized in Figure 2 and Table 1. These calculations were carried out with the Gaussian 98 and 03 program suites. ${ }^{49,50}$ On the basis of comparison with measured vibrational frequencies for the reactants and products, the ab initio frequencies have been scaled by a factor of 0.9597 , to account for anharmonicity. This factor is close to the value of 0.955 derived for a variety of halomethanes and halocarbon radicals. ${ }^{51,52}$ The intrinsic reaction coordinate was explored in the neighborhood of the TS, and in the neighborhood of the complex and reactants the reaction coordinate was defined directly in terms of the length of the newly forming $\mathrm{C}-\mathrm{H}$ bond, $r(\mathrm{C}-\mathrm{H})$, held constant in relaxed scans of the PES. The QCISD/ 6-311G $(\mathrm{d}, \mathrm{p})$ energy relative to reactants is displayed as a function of $r(\mathrm{C}-\mathrm{H})$ in Figure 3.

Next, more refined energies were obtained at points along the QCISD/6-311G $(\mathrm{d}, \mathrm{p})$ reaction coordinate by means of coupled cluster calculations. The Molpro 2002.6 program $^{53}$ was used to compute spin-restricted $\operatorname{CCSD}(\mathrm{T})$ energies with correlation consistent basis sets. The cc-pVnZ-PP basis sets of Peterson et al. with $n=2-4$ were employed. ${ }^{54}$ These basis sets treat the inner $1 \mathrm{~s}, 2 \mathrm{~s}$, and $2 \mathrm{p}$ electrons of bromine as a pseudopotential, which partly accounts for relativistic effects. The $4 \mathrm{~s}$ and $4 \mathrm{p}$ orbitals in bromine, and the $1 \mathrm{~s}$ hydrogen orbitals and the $2 \mathrm{~s}$ and $2 \mathrm{p}$ orbitals in carbon, and all the virtual orbitals, were included in the correlation treatment. Results for the stationary points are included in Table 1 . These data were employed in an extrapolation of energy as a function of $n$ in the form

$$
E_{n}=E_{\mathrm{CBS}}+A \exp (-B n)
$$

to obtain the energy at the infinite $n$ or complete basis set limit, $E_{\mathrm{CBS}}$. These results are plotted in Figure 2.

The overall computed reaction enthalpy, combined with an empirical spin-orbit correction for atomic $\mathrm{Br}$, is $-71.9 \mathrm{~kJ}$ $\mathrm{mol}^{-1}$, which is in excellent accord with the JANAF value of $-69.4 \mathrm{~kJ} \mathrm{~mol}^{-1}{ }^{25}$ Any errors in the computed bond strengths are therefore well-balanced and should largely cancel along the reaction coordinate. An indication that errors in individual bond strengths are likely to be small comes from the work of Feller et al. ${ }^{55}$ Methods similar to those applied here yielded errors in the atomization enthalpies for $\mathrm{HBr}$ and $\mathrm{CH}_{3} \mathrm{Br}$ of 0.5 and 2.5 $\mathrm{kJ} \mathrm{mol}^{-1}$, respectively, an average of $0.6 \mathrm{~kJ} \mathrm{~mol}^{-1}$ per bond.

Figure 3 shows that the TS is located accurately by QCISD theory with a medium sized basis set, although its energy is too positive compared to the results obtained from inclusion of the triples contributions to the correlation energy at the infinite basis set limit. The position of the minimum shifts to shorter $r(\mathrm{C}-\mathrm{H})$ with the higher level calculations. When zero-point vibrational energies are included, the complex remains bound, by about $4 \mathrm{~kJ} \mathrm{~mol}^{-1}$ at $0 \mathrm{~K}$, and the TS lies below the reactants, by $2.3 \mathrm{~kJ} \mathrm{~mol}^{-1}$.

Calculations of the Rate Constants for Reaction 1a and 1b Using the Modified Transition State Theory. The structural and other parameters of the transition states for reaction 1 listed in Table 2 were used to calculate the rate constant based on the modified transition state theory using the set of expressions E39. The entrance parts of the PESs including zero-point vibrational energy were carefully investigated. No entrance barrier for reaction la was found within the digital noise of the calculations (about $0.1 \mathrm{~kJ} \mathrm{~mol}^{-1}$ ). The following procedure was then used to evaluate the effective rotational constant for the centrifugal barriers, $B_{1, \text { eff }}(H)$. Estimates show that only quantum numbers $J<40$ play roles in the corrections. Therefore, the potential + centrifugal energy was calculated for discrete $J(5,10,20,30$, 40 , and 50). The centrifugal barrier height was plotted vs $J(J+1)$. A slightly curved plot was fitted with a linear dependence, which yields the effective rotational constant used in the subsequent calculations. The deviation of the fit from the actual energies was small, less than $0.1 \mathrm{~kJ} \mathrm{~mol}^{-1}$. In the case of the D-analogue, reaction $1 \mathrm{~b}$, a small entrance "barrier" was recognized in the entrance valley, with the top almost exactly at the reactants level. Application of the procedure outlined above led to location of 
TABLE 2: Parameters of the Reactants, Transition States and the Entrance Structures Used in the Calculations

\begin{tabular}{|c|c|c|c|c|c|c|}
\hline species & frequencies $/ \mathrm{cm}^{-1}$ & $B / G H z$ & $A / G H z$ & $\begin{array}{c}\text { energy relative } \\
\text { to reactants } \\
\mathrm{kJ} \mathrm{mol}^{-1}\end{array}$ & $\begin{array}{l}\text { entrance barrier, } \\
\qquad V_{10} / \mathrm{kJ} \mathrm{mol}^{-1}\end{array}$ & $\begin{array}{c}\text { effective rotational } \\
\text { constant, }{ }^{b} \\
B_{1, \text { eff }} / \mathrm{GHz}\end{array}$ \\
\hline $\mathrm{H}^{79} \mathrm{Br}$ & 2581 & 253.17 & & & & \\
\hline $\mathrm{D}^{79} \mathrm{Br}$ & 1837 & 128.3 & & & & \\
\hline $\mathrm{CH}_{3}$ & $3176(2), 3002,1378(2), 415$ & 285.0 & 142.5 & & & \\
\hline $\begin{array}{l}\text { transition state } \\
\qquad \mathrm{H}_{3} \mathrm{C} \cdots \mathrm{H} \cdots \mathrm{Br}^{\ddagger}\end{array}$ & $\begin{array}{l}3146(2), 2984,1385(2), 1236,687(2) \\
\quad 683,287(2), 497 \mathrm{i}\end{array}$ & 3.931 & 145.3 & -2.30 & & \\
\hline $\begin{array}{l}\text { transition state } \\
\qquad \mathrm{H}_{3} \mathrm{C} \cdot \cdots \mathrm{D} \cdots \mathrm{Br}^{\ddagger}\end{array}$ & $\begin{array}{l}3146(2), 2984,1386(2), 1042,630,574(2), \\
\quad 245(2), 455 \mathrm{i}\end{array}$ & 3.901 & 145.3 & -1.17 & & \\
\hline $\begin{array}{c}\text { entrance structure } \\
\mathrm{H}_{3} \mathrm{C} \cdots \mathrm{H}-\mathrm{Br}\end{array}$ & & & & & 0 & 1.008 \\
\hline $\begin{array}{c}\text { entrance structure } \\
\mathrm{H}_{3} \mathrm{C} \cdots \mathrm{D}-\mathrm{Br}\end{array}$ & & & & & 0 & 0.903 \\
\hline
\end{tabular}

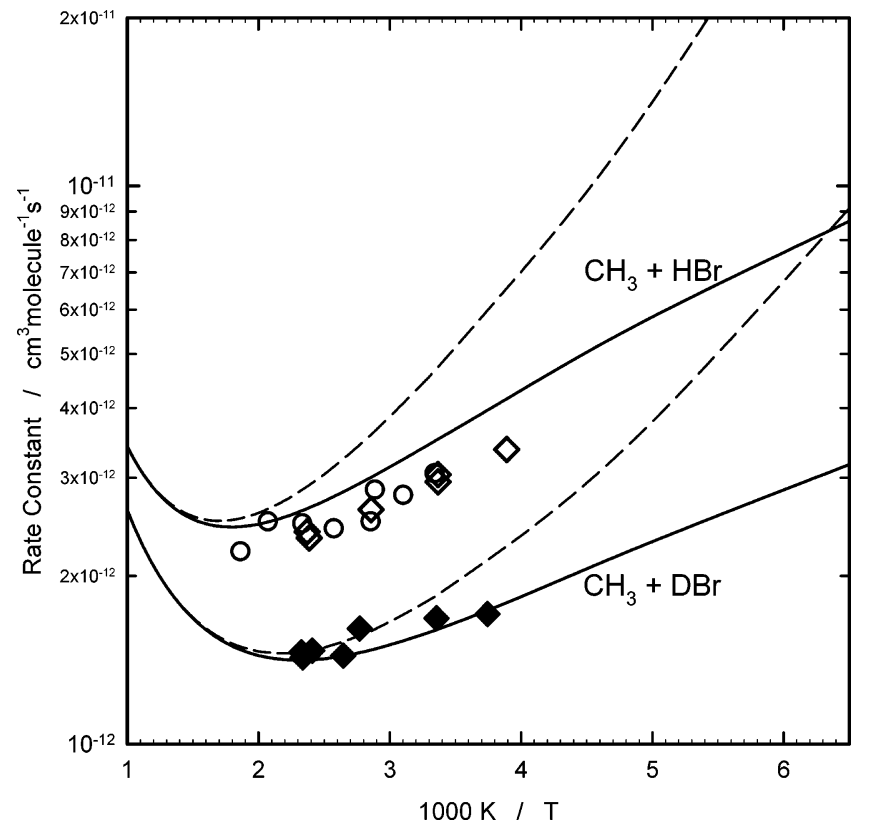

Figure 4. Rate constants for reactions $\mathrm{CH}_{3}+\mathrm{HBr}$ (1a) and $\mathrm{CH}_{3}+$ $\mathrm{DBr}(1 \mathrm{~b})$. Points: experiment. Open circles and diamonds: reaction 1a, refs 1 and 2, respectively. Filled diamonds: reaction 1b, ref 2. Solid lines: calculations using the modified transition state theory (expressions E39b-E39j) using the theoretical ab initio data without adjustments or fitting parameters. Dashed lines represent the results of the TST theory (with tunneling) with formally substituted negative barrier heights (E39a).

all centrifugal barriers at the same reaction coordinate; therefore the plot of the barrier energy vs $J(J+1)$ was perfectly linear. The values of the effective rate constants for the entrance centrifugal barriers obtained in this way are listed in Table 2.

The results of the calculations are shown in Figure 4. The negative temperature dependences of the rate constants for both (1a) and (1b), as well as the absolute value of the rate constant for reaction $1 \mathrm{~b}$, are reproduced almost perfectly. The deviation of the calculated and experimental rate constants for reaction 1a is on average $10 \%$, within the likely accuracy of the experiments. The isotope substitution effect $k_{\mathrm{H}} / k_{\mathrm{D}}$ is reproduced with an accuracy of $10 \%$. It should be stressed that, as no adjustments of any parameters has been made in these calculations, the result is $100 \%$ ab initio. The agreement between the theory and the experiment is considered very good, and might be somewhat fortuitous. An arbitrary adjustment of the barrier position from -2.3 to $-1.9 \mathrm{~kJ} \mathrm{~mol}^{-1}$ brings the calculations into perfect agreement for reaction $1 \mathrm{a}$, with a necessary deviation of the calculations from the experiment for reaction $1 b$ by about
$10 \%$. However, variations of the barrier position within a reasonable range do not change either the isotope substitution effect or the negative temperature dependence. The scale of the quantum effects (tunneling + reflection) is ca. $18 \%$ at $150 \mathrm{~K}$ and $7 \%$ at $500 \mathrm{~K}$ for reaction $1 \mathrm{a}$.

In addition to the results of the modified transition state theory, the predictions of standard TST with the same tunneling correction, where the negative barrier is formally used as the activation barrier, are shown as dotted lines in Figure 4. As was stated before, the modified TST predicts smaller rate constants and lesser negative temperature dependence than the classical TST with formal negative barriers. This comparison is of interest from another point of view-that of possible pressure dependence of reactions with negative barriers. If a surrounding medium is provided that ensures fast energy relaxation on the time scale of passing from the entrance barrier to the transition state, then the classical TST should become applicable. Such a surrounding could be a high pressure bath gas, a supercritical fluid, or a liquid solvent. Therefore, the difference between the dashed lines and solid lines represents the predicted pressure effect on the rate of reaction 1 . As can be seen in Figure 4, only a relatively minor pressure effect is expected at room temperature (ca. 30\%). It is interesting to note that a similarly sized increase in the rate constant of reaction 1a in He over the pressure range 1-100 bar was observed but was not reliably identified due to large experimental errors at elevated pressures. ${ }^{56}$ Large pressure effects are expected at lower temperatures. For example, at $200 \mathrm{~K}$ the expected increase of the rate constant in conditions of fast energy relaxation is about a factor of 2.3 for reaction $1 \mathrm{a}$.

In view of the results of the theoretical treatment of reactions with negative barriers outlined above, it is necessary to comment on the widespread application of canonical variational transition state theory ${ }^{33-44}$ and some of its variants to the so-called "barrierless" reactions. These theories are based on the concept of flux minimization, which (in the canonical form) can be reformulated as a requirement of maximum free energy. However, this can be justified only when the energy of the system in the bottleneck position is positive. ${ }^{18,19}$ In the case of barrierless reactions, the position of the bottleneck is expected at negative energies. Although the free energy when formally calculated could still exhibit an extremum (maximum) at the bottleneck position above the free energy of the reactants due to the entropic contribution, the very concept of the free energy is not applicable. As was shown in the preceding sections, for negative barriers the rates cannot be expressed via the partition function of the transition state. Therefore, the formal application of the classical canonical TST or any of its variants (except for the Improved Canonical Variational Theory ${ }^{18,19}$ ) to reactions 
where the bottleneck has energy below the ground state energy is not justified. For such reactions, either the Improved Canonical Variational Theory ${ }^{18,19}$ or modified Transition State Theory (in cases when the bottleneck position is well described by a local maximum on the potential energy curve) should be used.

\section{Conclusions}

The simplest reaction of the type $\mathrm{R}+\mathrm{HX}$, reaction of $\mathrm{CH}_{3}$ radical with $\mathrm{HBr}$, that exhibits a negative temperature dependence was successfully treated on the basis of the modified transition state theory and accurate ab initio calculations. The negative temperature dependence, the absolute values of the rate constant, as well as the isotope substitution effect are reproduced with a good accuracy without any adjustment or fitting parameters. The developed modified transition state theory is based on the general assumptions of the classical TST and does not require a long-lived statistical complex to explain the experimental observations. The theory has a wider range of applications than the reactions of free radicals with halogens and hydrogen halides, and can be used for other barrierless reactions (such as free radical recombination, etc.). One of the important conclusions is that application of traditional canonical variational TST to reactions in which the bottleneck has a negative energy with respect to the reactants, in the gas phase at moderate pressures, should be reconsidered.

Acknowledgment. P.M. thanks the R.A. Welch Foundation (Grant B-1174) for support. Computer resources were provided in part by the Materials Directorate (Air Force Research Laboratory) at Wright-Patterson AFB and the National Center for Supercomputing Applications (Grant CHE 000015N). Additional facilities were provided on the Research Cluster operated by UNT Academic Computing Services and on a cluster purchased with NSF support (Grant CHE 0342824).

\section{Appendix 1}

Derivation for Bimolecular Reaction Using an Artificial Nonbonded Complex. For bimolecular reaction E1 let us introduce an artificial "complex" of molecules A and B at arbitrary separation but before the entrance to the reaction valley:

$$
\mathrm{A}+\mathrm{B} \Leftrightarrow \mathrm{C} \rightarrow \mathrm{AB}^{\ddagger} \rightarrow \text { products }
$$

The complex is assumed to be in microcanonical equilibrium (not the steady state) with the reactants at any available energy, the reactants being in the canonical equilibrium state. This is consistent with the general assumptions of the transition state theory. ${ }^{30}$ The total concentration of the complexes is then

$$
[\mathrm{C}]=\left(Q_{\mathrm{C}} / Q_{\mathrm{A}} Q_{\mathrm{B}}\right)[\mathrm{A}][\mathrm{B}]
$$

The reaction rate is the rate constant of unimolecular transformation of "complexes" into the products, $k_{\mathrm{C}}$, multiplied by the concentration of the complexes:

$$
\text { rate }=k[\mathrm{~A}][\mathrm{B}]=k_{\mathrm{C}}[\mathrm{C}]=k_{\mathrm{C}}\left(Q_{\mathrm{C}} / Q_{\mathrm{A}} Q_{\mathrm{B}}\right)[\mathrm{A}][\mathrm{B}]
$$

Therefore, the rate constant of reaction E1 is obtained from the rate constant of the unimolecular transformation of the complex by multiplication by the ratio of the partition functions:

$$
k=k_{\mathrm{C}}\left(Q_{\mathrm{C}} / Q_{\mathrm{A}} Q_{\mathrm{B}}\right)
$$

Because statistical theories lead to $k_{\mathrm{C}}$ inversely proportional to
$Q_{\mathrm{C}}$, the partition functions of the artificial "complex" cancels out in (E44), so that the specific parameters of the complex are not required.

\section{Appendix 2}

Evaluation of the Generalized Transmission Function, Tr$(\boldsymbol{a}, \boldsymbol{b}, \boldsymbol{x})$. The generalized transmission function, defined as below, can be expressed through the hypergeometric function ${ }_{2} F_{1}: 57$

$$
\begin{aligned}
& \operatorname{Tr}(a, b, x)=\int_{-\infty}^{x} \frac{\exp (b \xi)}{1+\exp (-a \xi)} \mathrm{d} \xi= \\
& \frac{\exp (b x)(1+\exp (a x))}{(a+b)(1+\exp (-a x))^{2}} F_{1}\left(1,(1+b / a),(2+b / a),\left(-\mathrm{e}^{a x}\right)\right)
\end{aligned}
$$

The hypergeometric function ${ }_{2} F_{1}$ can be represented as a series. ${ }^{58}$ For $|z|<1$,

$$
\begin{aligned}
&{ }_{2} F_{1}(\alpha, \beta, \gamma, z)=1+\frac{\alpha \beta}{\gamma} \frac{z}{1 !}+\frac{\alpha(\alpha+1) \beta(\beta+1)}{\gamma(\gamma+1)} \frac{z^{2}}{2 !}+ \\
& \frac{\alpha(\alpha+1)(\alpha+2) \beta(\beta+1)(\beta+2)}{\gamma(\gamma+1)(\gamma+2)} \frac{z^{3}}{3 !}+\ldots
\end{aligned}
$$

Substituting $\alpha=1, \beta=1+b / a, \gamma=2+b / a, \mathrm{z}=-\exp (a x)$ as in (E45), the series is simplified:

$$
\begin{aligned}
& { }_{2} F_{1}\left(1,(1+b / a),(2+b / a),\left(-\mathrm{e}^{a x}\right)\right) \\
& =1+\frac{(1+b / a)}{(2+b / a)} \frac{1}{1 !}\left(-\mathrm{e}^{a x}\right)+\frac{2(1+b / a)(2+b / a)}{(2+b / a)(3+b / a)} \frac{1}{2 !}\left(\mathrm{e}^{2 a x}\right)+ \\
& \frac{6(1+b / a)(2+b / a)(3+b / a)}{(2+b / a)(3+b / a)(4+b / a)} \frac{1}{3 !}\left(-\mathrm{e}^{3 a x}\right) \ldots \\
& =1-\frac{(a+b)}{(2 a+b)} \mathrm{e}^{a x}+\frac{(a+b)}{(3 a+b)} \mathrm{e}^{2 a x}-\frac{(a+b)}{(4 a+b)} \mathrm{e}^{3 a x}+ \\
& \frac{(a+b)}{(5 a+b)} \mathrm{e}^{4 a x}+\ldots \text { (E47) }
\end{aligned}
$$

Expression E47 is valid for $|z|<1$ (i.e., $x<0$ ). For positive $x$, a transformation $z \rightarrow 1 / z$, is used:

$$
\begin{array}{r}
{ }_{2} F_{1}(\alpha, \beta, \gamma, z)=\left[\frac{\Gamma(\gamma) \Gamma(\beta-\alpha)}{\Gamma(\beta) \Gamma(\gamma-\alpha)}\right](-z){ }_{2}^{-\alpha} F_{1}(\alpha, \alpha+1-\gamma, \alpha+1- \\
\left.\beta, \frac{1}{z}\right)+\left[\frac{\Gamma(\gamma) \Gamma(\alpha-\beta)}{\Gamma(\alpha) \Gamma(\gamma-\beta)}\right](-z)^{-\beta}{ }_{2} F_{1}\left(\beta, \beta+1-\gamma, \beta+1-\alpha, \frac{1}{z}\right)
\end{array}
$$

Applying the series E46 to the hypergeometric functions in (E48), after some transformations, for $x>0$, the following expression is obtained:

$$
\begin{aligned}
& { }_{2} F_{1}\left(1,(1+b / a),(2+b / a),\left(-\mathrm{e}^{a x}\right)\right)=\frac{(a+b)}{b} \mathrm{e}^{-a x} \times \\
& \left\{1+\frac{b}{a-b} \mathrm{e}^{-a x}-\frac{b}{2 a-b} \mathrm{e}^{-2 a x}+\frac{b}{3 a-b} \mathrm{e}^{-3 a x}+\ldots\right\}+ \\
& \Gamma(2+b / a) \Gamma(-b / a) \mathrm{e}^{-(a+b) x}
\end{aligned}
$$

\section{References and Notes}

(1) Seakins, P. W.; Pilling, M. J.; Niiranen, J. T.; Gutman, D.; Krasnoperov, L. N. J. Phys. Chem. 1992, 96, 9847.

(2) Nicovich, J. M.; Van Dijk, C. A.; Kreutter, K. D.; Wine, P. H. J. Phys. Chem. 1991, 95, 9890.

(3) Baulch, D. L.; Drysdale, D. D. Combust. Flame 1974, 23, 215. 
(4) Perry, R. A.; Atkinson, R.; Pitts, J. N., Jr. J. Chem. Phys. 1977, 67, 5577.

(5) Smith, I. W. M.; Zellner, R. J. Chem. Soc., Faraday Trans. 2 1973, 69, 1617. 28.

6) Patrick, R.; Barker, J. R.; Golden, D. M. J. Phys. Chem. 1984, 88 ,

(7) Smith, I. W. M.; Herbst, E.; Chang, Q. Mon. Not. R. Astronom. Soc. 2004, 350, 323.

(8) Mozurkewich, M.; Benson, S. W. J. Phys. Chem. 1984, 88, 6429

(9) Benson, S. W.; Dobis, O. J. Phys. Chem. A 1998, 102, 5175.

(10) Mozurkewich, M.; Benson, S. W. J. Phys. Chem. 1984, 88, 6429

(11) Russell, J. J.; Seetula, J. A.; Gutman, D. J. Am. Chem. Soc. 1988, 110,3092 .

(12) Niiranen, J. T.; Gutman, D.; Krasnoperov, L. N. J. Phys. Chem. 1992, 96, 5881

(13) Kalinovski, I. J.; Gutman, D.; Krasnoperov, L. N.; Goumri, A.;

Yuan, W.-J.; Marshall, P. J. Phys. Chem. 1994, 98, 9551.

(14) Seetula, J. A. Phys. Chem. Chem. Phys. 2002, 4, 455.

(15) Benson, S. W.; Dobis, O. J. Phys. Chem. A 1998, 102, 5175.

(16) Chen, Y.; Rauk, A.; Tschuikow-Roux, E. J. Phys. Chem. 1991, 95, 9900 .

(17) Chen, Y.; Tschuikow-Roux, E. J. Phys. Chem. 1993, 97, 3742

(18) Garrett, B. C.; Truhlar, D. G. J. Phys. Chem. 1980, 84, 805.

(19) Garrett, B. C.; Truhlar, D. G.; Grev, R. S.; Magnuson, A. W. J. Phys. Chem. 1980, 84, 1730

(20) Isaacson, A. D.; Sund, M. T.; Ray, S. N.; Truhlar, D. G. J. Chem. Phys. 1985, 82, 1338 .

(21) Chen, Y.; Rauk, A.; Tschuikow-Roux, E. J. Phys. Chem. 1991, 95, 9900 .

(22) Benson, S. W.; Dobis, O. J. Phys. Chem. A 1998, 102, 5175

(23) Yu, H.-G.; Nyman, G. J. Phys. Chem. A 2001, 105, 2240. 71.

(24) Guha, S.; Francisco, J. S. J. Mol. Struct. (THEOCHEM) 2001, 573 ,

(25) NIST-JANAF Thermochemical Tables, 4th ed.; Chase, M. W., Jr., Ed.; American Chemical Society and the American Institute of Physics: Washington, DC, and Woodbury, NY, 1998.

(26) Espinosa-Garcia, J. J. Chem. Phys. 2002, 117, 2076.

(27) Sheng, L.; Li, Z.-S.; Liu, J.-Y.; Sun, C.-C. J. Chem. Phys. 2003, 119,10585 .

(28) Eyring, H. J. Chem. Phys. 1935, 3, 107

(29) Evans, M. J.; Polyani, M. Trans. Faraday Soc. 1935, 31, 875.

(30) Truhlar, D. G.; Garrett, B. C.; Klippenstein, S. J. J. Phys. Chem. 1996, $100,12771$.

(31) Wigner, E. Trans. Faraday Soc. 1938, 34, 29

(32) Tolman, R. C. The Principles of Statistical Mechanics; Dover: New York, 1979.

(33) Fowler, R. H.; Guggenheim, E. A. Statistical Thermodynamics; University Press: Cambridge, England, 1939.

(34) Truhlar, D. G.; Garrett, B. C. Annu. Rev. Phys. Chem. 1984, 35, 159.

(35) Bishop, D. M.; Laidler, K. J. Trans. Faraday Soc. 1970, 66, 1685

(36) Keck, J. C. J. Chem. Phys. 1967, 13, 85.

(37) Quack, M.; Troe, J. Ber. Bunsen-Ges. Phys. Chem. 1974, 78, 240.

(38) Pechukas, P. Annu. Rev. Phys. Chem. 1981, 32, 159.

(39) Smith, I. W. M. Kinetics and Dynamics of Elementary Gas Reactions; Butterworth: Boston, 1980

(40) Wardlaw, D. M.; Markus, R. A. Adv. Chem. Phys. 1987, 70, 231

(41) Robertson, S. H.; Wagner, A. F.; Wardlaw, D. M. J. Chem. Phys. 1995, 103, 2917.
(42) Quack, M.; Troe, J. Ber. Bunsen-Ges. Phys. Chem. 1975, 79, 170

(43) Quack, M.; Troe, J. Ber. Bunsen-Ges. Phys. Chem. 1977, 81, 329.

(44) Klippenstein, S. J. Chem. Phys. Lett. 1990, 170, 71.

(45) Nikitin, E. E. Theory of Elementary Atomic and Molecular Processes in Gases; Clarendon: Oxford, U.K., 1974.

(46) Holbrook, K. A.; Pilling, M. J.; Robertson, S. H. Unimolecular Reactions; John Wiley \& Sons: New York, 1996.

(47) Steinfeld, J. I.; Francisco, J. S.; Hase, W. L. Chemical Kinetics and Dynamics; Prentice Hall: Englewood Cliffs, NJ, 1989.

(48) Garrett, B. C.; Truhlar, D. G. J. Phys. Chem. 1979, 83, 200.

(49) Frisch, M. J.; Trucks, G. W.; Schlegel, H. B.; Scuseria, G. B.; Robb M. A.; Cheeseman, J. R.; Zakrzewski, V. G.; Montgomery, J. A., Jr.; Stratmann, R. E.; Burant, J. C.; Dapprich, S.; Millam, J. M.; Daniels, A. D.; Kudin, K. N.; Strain, M. C.; Farkas, O.; Tomasi, J.; Barone, V.; Cossi, M.; Cammi, R.; Mennucci, B.; Pomelli, C.; Adamo, C.; Clifford, S.; Ochterski, J.; Petersson, G. A.; Ayala, P. Y.; Cui, Q.; Morokuma, K.; Malick, D. K.; Rabuck, A. D.; Raghavachari, K.; Foresman, J. B.; Cioslowski, J.; Ortiz, J. V.; Baboul, A. G.; Stefanov, B. B.; Liu, G.; Liashenko, A.; Piskorz, P.; Komaromi, I.; Gomperts, R.; Martin, R. L.; Fox, D. J.; Keith, T.; AlLaham, M. A.; Peng, C. Y.; Nanayakkara, A.; Challacombe, M.; Gill, P M. W.; Johnson, B.; Chen, W.; Wong, M. W.; Andres, J. L.; Gonzalez, C.; Head-Gordon, M.; Replogle, E. S.; Pople, J. A. Gaussian 98, revision A.9; Gaussian, Inc.: Pittsburgh, PA, 1998.

(50) Frisch, M. J.; Trucks, G. W.; Schlegel, H. B.; Scuseria, G. E.; Robb M. A.; Cheeseman, J. R.; Montgomery, J. A., Jr.; Vreven, T.; Kudin, K. N.; Burant, J. C.; Millam, J. M.; Iyengar, S. S.; Tomasi, J.; Barone, V.; Mennucci, B.; Cossi, M.; Scalmani, G.; Rega, N.; Petersson, G. A. Nakatsuji, H.; Hada, M.; Ehara, M.; Toyota, K.; Fukuda, R.; Hasegawa, J.; Ishida, M.; Nakajima, T.; Honda, Y.; Kitao, O.; Nakai, H.; Klene, M.; Li, X.; Knox, J. E.; Hratchian, H. P.; Cross, J. B.; Adamo, C.; Jaramillo, J.; Gomperts, R.; Stratmann, R. E.; Yazyev, O.; Austin, A. J.; Cammi, R.; Pomelli, C.; Ochterski, J. W.; Ayala, P. Y.; Morokuma, K.; Voth, G. A.; Salvador, P.; Dannenberg, J. J.; Zakrzewski, V. G.; Dapprich, S.; Daniels, A. D.; Strain, M. C.; Farkas, O.; Malick, D. K.; Rabuck, A. D. Raghavachari, K.; Foresman, J. B.; Ortiz, J. V.; Cui, Q.; Baboul, A. G.; Clifford, S.; Cioslowski, J.; Stefanov, B. B.; Liu, G.; Liashenko, A.; Piskorz, P.; Komaromi, I.; Martin, R. L.; Fox, D. J.; Keith, T.; Al-Laham, M. A.; Peng, C. Y.; Nanayakkara, A.; Challacombe, M.; Gill, P. M. W.; Johnson, B.; Chen, W.; Wong, M. W.; Gonzalez, C.; Pople, J. A. Gaussian 03, revision B.04; Gaussian Inc.: Pittsburgh, PA, 2003.

(51) Schwartz, M.; Marshall, P. J. Phys. Chem. A 1999, 103, 7900.

(52) Schwartz, M.; Peebles, L. R.; Berry, R. J.; Marshall, P. J. Chem. Phys. 2003, 118, 557 .

(53) Knowles, P. J.; Lindh, R.; Schutz, M.; Celani, P.; Korona, T.; Manby, F. R.; Rauhut, G.; Amos, R. D.; Bernhardsson, A.; Berning, A.; Cooper, D. L.; Deegan, M. J. O.; Dobbyn, A. J.; Eckert, F.; Hampel, C.; Hetzer, G.; Lloyd, A. W.; McNicholas, S. J.; Meyer, W.; Mura, M. E.; Nicklass, A.; Palmieri, P.; Pitzer, R.; Schumann, U.; Stoll, H.; Stone, A. J.; Tarroni, R.; Thorsteinsson, T. Molpro, revision 2002.6; 2002

(54) Peterson, K. A.; Figgen, D.; Goll, E.; Stoll, H.; Dolg, M. J. Chem. Phys. 2003, 119, 11113.

(55) Feller, D.; Peterson, K. A.; De Jong, W. A.; Dixon, D. A. J. Chem. Phys. 2003, 118,3510

(56) Krasnoperov, L. N.; Mehta, K. J. Phys. Chem. A 1999, 103, 8008.

(57) http://integrals.com/index.cgi.

(58) http://mathworld.wolfram.com/HypergeometricFunction.html. 